\title{
The effect of myocardial fibrosis on left ventricular torsion and twist in patients with non-ischemic dilated cardiomyopathy
}

\author{
Tansu Karaahmet ${ }^{1}$, Emre Gürel ${ }^{2}$, Kursat Tigen ${ }^{3}$, Ahmet Güler ${ }^{3}$, \\ Cihan Dündar ${ }^{3}$, Hakan Fotbolcu ${ }^{4}$, Yelda Basaran ${ }^{5}$ \\ ${ }^{1}$ Department of Cardiology, Acibadem University, Istanbul, Turkey \\ ${ }^{2}$ Department of Cardiology, Ordu State Hospital, Ordu, Turkey \\ ${ }^{3}$ Department of Cardiology, Kartal Koşuyolu Heart and Research Hospital, Istanbul, Turkey \\ ${ }^{4}$ Department of Cardiology, Delta Hospital, Istanbul, Turkey \\ ${ }^{5}$ Department of Cardiology, Marmara University Hospital, Istanbul, Turkey
}

\begin{abstract}
Background: Left ventricular $(L V)$ rotation, twist, and torsion are important aspects of the cardiac performance. Myocardial fibrosis can be identified as the late gadolinium enhancement (LGE) in cardiac magnetic resonance (CMR). In this study, we investigated the association between myocardial fibrosis and LV rotational parameters in patients with nonischemic dilated cardiomyopathy (NDC).
\end{abstract}

Methods: Twenty-two NDC patients were enrolled. LV dimensions, volumes and ejection fraction (EF) were measured, conventional tissue Doppler imaging data was acquired. Speckle tracking imaging was performed to measure LV deformation, LV rotational parameters. Blood samples were obtained for NT-proBNP. Late gadolinium enhanced cardiac magnetic resonance (LGE-CMR) was used to assess cardiac fibrosis index.

Results: Myocardial deformation was similar between LGE + and LGE- groups. LGE+ patients have significantly higher basal and lower apical systolic rotation, lower twist and torsion when compared to LGE-patients. However, untwisting rate was similar between the groups. Torsion was significantly correlated with LVEF and MR-index. Patients with reversed apical systolic rotation had significantly greater NT-proBNP values, basal systolic rotation and significantly lower apical systolic rotation, torsion, and MR-index.

Conclusions: Cardiac fibrosis index is closely related with myocardial torsion and LV systolic function and may be used for the evaluation of cardiac condition. Reversed apical systolic rotation indicated more extensive cardiac fibrosis as it may reflect severe LV dyssynchrony and poor LV performance. (Cardiol J 2013; 20, 3: 276-286)

Key words: dilated cardiomyopathy, magnetic resonance, echocardiography

Address for correspondence: Dr Emre Gürel, Bahçelievler Mh. 293.Sk. Gürsoy-Varol Sitesi, B-blok, No: 4/13, Ordu, Turkey, tel: +90 533 4232151, fax: +90 452 2343232, e-mail: emregurelctf@yahoo.com

Received: 02.10 .2012

Accepted: 24.11.2012 


\section{Introduction}

Heart failure remains one of the major public health problems in developed countries [1,2]. During the development of congestive heart failure in patients with dilated cardiomyopathy (DC), the left ventricle (LV) undergoes complex changes in geometry, accompanied by alteration of the LV chamber and myocardial systolic and/or diastolic function. $\mathrm{LV}$ ejection fraction (EF) is the most commonly used parameter for the severity of LV systolic dysfunction in patients with congestive heart failure and has been used as a prognostic marker [3, 4]. $\mathrm{LV}$ rotation, twist, and torsion are also important aspects of the cardiac performance. Rotation of the $\mathrm{LV}$ apex relative to the base is related to the ventricular efficiency. Potential energy stored by LV twist during the systolic phase is rapidly released during LV untwisting and constitutes an important determinant of diastolic suction and function [5]. Two-dimensional speckle tracking imaging (2D-STI) is a novel ultrasound technique for quantification of LV regional deformation, myocardial rotation, torsion, twist and untwisting [6,7]. This technique has been validated by tagged magnetic resonance imaging, which is currently considered gold standard for the assessment of myocardial rotational parameters [8, 9]. Myocardial fibrosis could be identified as the late gadolinium enhancement (LGE) areas in cardiac magnetic resonance (CMR) studies $[10,11]$. Recent studies reported that LGE was associated with adverse prognosis in patients with non-ischemic dilated cardiomyopathy (NDC) $[11,12]$. However, there is limited data about the association between myocardial fibrosis and LV rotational parameters in NDC patients, which is the main purpose of this investigation.

\section{Methods}

\section{Study population}

Twenty-two patients (17 men and 5 women; average age $47 \pm 12$ years, range $27-74$ ) with NDC and sinus rhythm were enrolled from the outpatient clinic of Koşuyolu Heart and Research Hospital, between September 2008 and June 2009. Patients with organic valvular heart disease, history of coronary artery disease (acute coronary syndrome, revascularization history, $>50 \%$ luminal narrowing in any of the coronary arteries), atrial fibrillation, permanent pacemakers, chronic kidney disease ( $>$ stage 3 kidney disease), LVEF $>40 \%$, and patients with inadequate quality for speckle-tracking analysis were not included. Functional capacities were assessed by New York Heart Association
(NYHA) classification, and physical examination findings with 12-lead electrocardiograms (ECG) were recorded. Blood samples for N-terminal pro-B-type natriuretic peptide (NT-proBNP) levels were obtained following a resting period after echocardiographic examinations. All patients underwent a routine coronary angiography and cardiac catheterization before enrolment to the study.

Written informed consent was obtained from all participants and the study was approved by the local ethics committee.

\section{Electrocardiographic examination}

Standard 12-lead ECG was acquired at a paper speed of $25 \mathrm{~mm} / \mathrm{s}$ and a scale of $10 \mathrm{~mm} / \mathrm{mV}$. Measurement of QRS duration (QRSd) and PR interval (PRd) (recorded from the surface leads demonstrating the greatest values) were performed by an experienced observer who was blind to the echocardiographic characteristics of the patients.

\section{Echocardiographic examinations}

Standard measurements. All echocardiographic measurements were performed using Vivid 7 (GE-Vingmed Ultrasound AS, Horten, Norway) with an M3S transducer. LV dimensions were measured by 2-dimensional M-mode echocardiography according to the guidelines of the American Society of Echocardiography [13]. LV volumes and EFs were calculated using the modified biplane Simpson's method (tracing LV end-diastolic and end-systolic cavities in apical 4- and 2-chamber views). Diastolic mitral inflow velocities were recorded by pulsed wave Doppler as previously described [14]. In apical 4-chamber view, sample volume was placed at the tips of the mitral leaflets and the following variables were measured: maximal flow velocity in early ( $\mathrm{E}$ wave) and late diastole (A wave), $\mathrm{E}$ wave deceleration time (EDT). The quantification of functional mitral regurgitation (MR) was performed using the proximal isovelocity surface area method [15]. Delta pressure/delta time (dp/dt) was calculated by the following formula; $\mathrm{dp} / \mathrm{dt}[\mathrm{mm} \mathrm{Hg} / \mathrm{s}]=32 /$ time [s] from the velocity of 1 to $3 \mathrm{~m} / \mathrm{s}$ achieved in the MR Doppler curve [16]. Tissue Doppler imaging (TDI) was performed in the apical views (4- and 3-chamber) in order to assess longitudinal velocities of the LV [17, 18]. Systolic velocity (Sm), early diastolic velocity (e'), and late diastolic velocity (a') were derived from the TDI study of the septal mitral annulus. The early diastolic mitral inflow velocity (E) and e' velocity ratio (E/e' ratio) were obtained. Sample volume was placed on the lateral tricuspid 


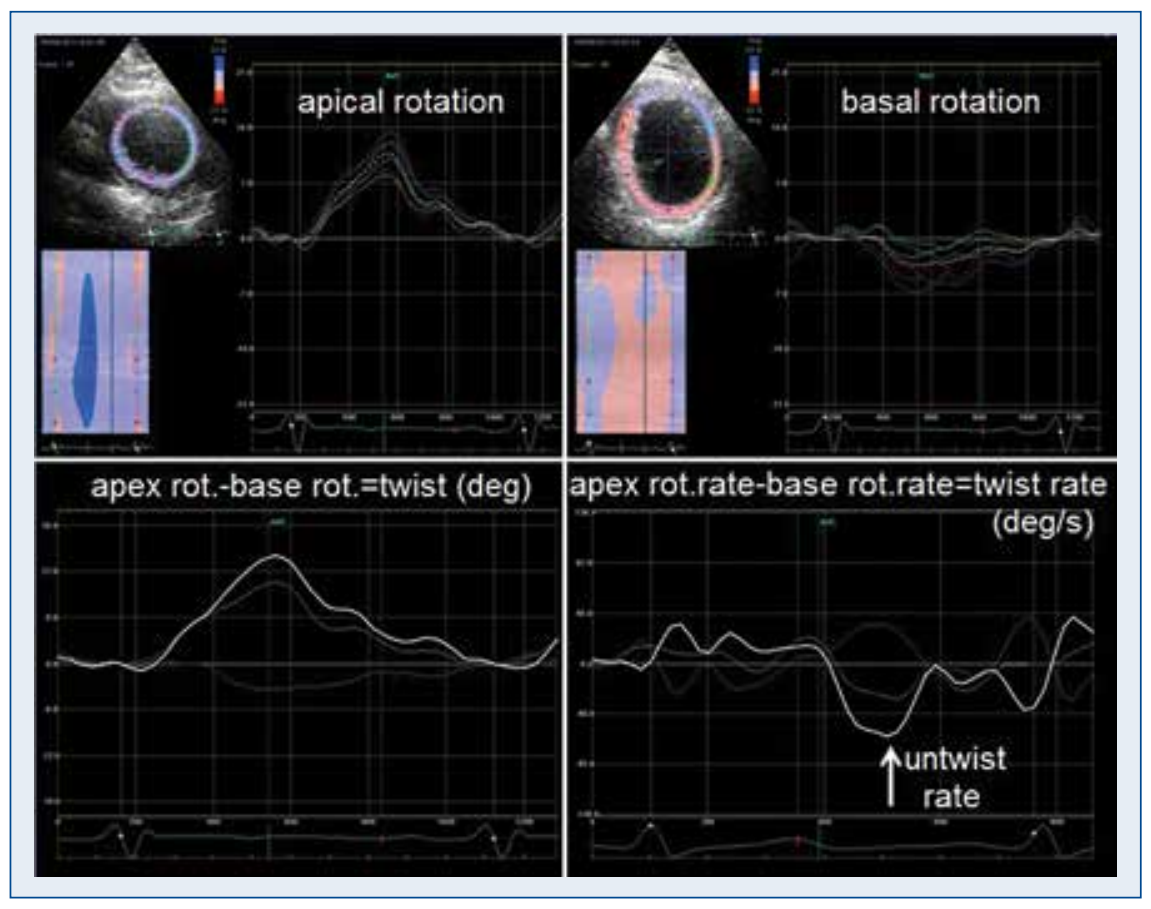

Figure 1. Left ventricular 'twist' and 'twist rate' were defined as the net difference of left ventricular (LV) peak systolic 'rotation' and 'rotation rate' between basal (clockwise) and apical (counterclockwise) short axis planes. Values were expressed in ' ${ }^{\prime \prime}$ and $\% / \mathrm{s}^{\prime}$, respectively. Negative values indicated clockwise while positive values indicated counterclockwise rotation. 'Untwist' was expressed as a diastolic angular motion of the LV, opposite to twist. 'Untwisting rate' $[\%$ s] was defined as the peak twist rate during early diastole.

annulus area and right ventricular systolic velocity (RVs) was measured to assess right ventricular systolic function.

Speckle tracking imaging. Two-dimensional, greyscale images (frame rate, $44-82$ frames/s) were acquired and recorded for offline analysis at a workstation (EchoPAC, GE, Horten, Norway). Apical 4- and 2-chamber views were used for longitudinal strain and strain rate analysis while parasternal short-axis (SAX) views at three levels (the base, the level of papillary muscles and the apex) were used for circumferential and radial strain, strain rate, twist and torsion analysis. End-systolic regions of interest were traced on the endocardial cavity (minimum cavity area) using a point-and-click approach with special care taken to adjust tracking of all endocardial segments. A second larger concentric circle was then automatically generated and manually adjusted near the epicardium. Speckle tracking automatically analyzed frame-by-frame movement of the speckles, over the cardiac cycle. Each image was divided into six standard segments, corresponding strain curves were obtained. The mean strain values were calculated for each SAX view, apical 4-chamber view and apical 2 -chamber view, as the sum of end-systolic strain values in 6 segments, divided by 6 . Global circumferential strain (GCS), global longitudinal strain (GLS), and global radial strain (GRS) were derived, using following formulas: GCS $=$ (mean basal circumferential strain + mean midcircumferential strain + mean apical circumferential strain) $/ 3$, GLS $=$ (mean longitudinal strain in the apical 4-chamber view + mean longitudinal strain in the apical 2 -chamber view) $/ 2$, and GRS $=$ (mean basal radial strain + mean midradial strain + mean apical radial strain)/3.

Two-dimensional speckle tracking analysis of parasternal SAX views at the base and at the apex revealed LV 'rotation' and 'rotation rate' curves as the angular displacement and the velocity of displacement of the LV around its central axis. Negative values indicated clockwise while positive values indicated counterclockwise rotation. LV 'twist' and 'twist rate' were defined as the net difference of LV peak systolic 'rotation' and 'rotation rate' between basal (clockwise) and apical (counterclockwise) short axis planes. Values were expressed in '0, and ' $\%$ ', respectively. 'Untwist' was expressed as a diastolic angular motion of the LV, opposite to twist. 'Untwisting rate' $[\%$ s] was defined as the peak twist rate during early diastole (Fig. 1). LV 
torsion was calculated as the net LV twist normalized with respect to ventricular diastolic longitudinal length between the $L V$ apex and the mitral plane $(\mathrm{LV}$ torsion $[\% \mathrm{~cm}]=[$ apical $\mathrm{LV}$ rotation - basal $\mathrm{LV}$ rotation]/LV diastolic longitudinal length) [19]. On the other hand, particular attention was paid to the direction of systolic apical rotation: normal (counterclockwise) or reversed (clockwise), which was documented for each patient.

Blood sampling for NT-proBNP. The blood samples for assessing plasma NT-proBNP levels were obtained by venipuncture and collected into ethylenediamine-tetraacetic acid tubes following the echocardiographic examinations. Each collected sample was centrifuged for $5 \mathrm{~min}$ at $2000 \mathrm{~g}$ and $4^{\circ} \mathrm{C}$ within $30 \mathrm{~min}$. The plasma was then separated off, frozen, and kept at $-80^{\circ} \mathrm{C}$ until the assessment time. NT-proBNP was measured with a proBNP assay on an Elecsys 2010 analyzer (Roche Diagnostics, GmbH, Mannheim, Germany). According to data provided by the manufacturer, the detection limit for this peptide is $5 \mathrm{pg} / \mathrm{mL}$.

Cardiac magnetic resonance imaging. CMR studies were obtained with 1.5-Tesla whole body scanner system (Magnetom, Avanto; Siemens, Medical Solutions, Erlangen, Germany) operating at a maximum gradient strength of $45 \mathrm{mT} \times \mathrm{m}^{-1}$ and slew rate of $200 \mathrm{~T} / \mathrm{m} / \mathrm{s}$, using six anterior channels and six posterior channels for data acquisition. An integrated parallel acquisition technique (iPAT) with an acceleration factor of 2 was used. Multislice short-axis cine imaging used ECG-triggered, steady-state free precession (slice thickness, $8 \mathrm{~mm}$; interslice gap 20\%) acquired from the atrioventricular ring to the apex. The CMR protocol included a functional study of the LV using an ECG-triggered breath-hold segmented steady-state free precession (SSFP; true fast imaging with steady-state free precession; FISP) cine sequence (temporal resolution, $44.25 \mathrm{~ms}$; echo time (TE) $1.25 \mathrm{~ms}$ flip angle, $52^{\circ}$ ) with a slice thickness of $8 \mathrm{~mm}$. The breath hold acquisition time was 6 to $9 \mathrm{~s}$. Depending on the field of view, the typical in-plane resolution was $1.9 \times 1.9 \mathrm{~mm}^{2}$ for all sequences. The total imaging time, including patient positioning, was 45-60 min. Dynamic studies were performed from the short axis plane at the basal, mid, and apical segments by using $0.1 \mathrm{mmoL} / \mathrm{kg}$ intravenous gadolinium contrast agent (Dotarem, Guerbet, France) given with a power injector (Medrad Stellant, PA, United States). Late enhancement scans were collected in three long-axis and all short-axis orientations by using a breath-hold ECG-triggered 2D inversion reco- very turbo FLASH sequence (TR/TE, 8/4 ms; flip angle, $25^{\circ}$ ). Images were acquired subsequently up to 15 min after injection. CMR images were re-analyzed and documented by using 17 -segment cardiac model that was recommended by the American Heart Association to improve standardization of the results [20]. LV was evaluated from the short axis images from basal, mid and apical segments. Basal and mid cavity were divided into 6 equal segments, and named as "anterior, anteroseptal, inferoseptal, inferior, posterolateral and anterolateral". Apical segment was divided into four sub-segments and named as "anterior, inferoseptal, inferior, and anterolateral". The apical cap was termed as "apex" and constituted the $17^{\text {th }}$ segment. The contrast-enhanced images were analyzed visually by 2 experienced observers who were blinded to other CMR, echocardiographic, and clinical data. LGE was rated by visual assessment and each segment was graded on a 2-point scale (segmental fibrosis score): $0=$ absence of LGE, $1=$ presence of LGE; with the method used by Kaandrop and coworkers owing to frequency of linear and patchy enhancement in patients with NDC [21]. Cardiac fibrosis index (MRI index) was calculated by, 17- (sum of fibrotic segments)/17 formula [22]. The patients were sub-grouped into two, based on the absence (LGE-) and presence (LGE+) of fibrosis in any of the left ventricular segments.

\section{Statistical analysis}

Statistical analysis was performed using a statistical software program (SPSS for Windows, version 15.0; SPSS Inc., Chicago, Illinois, USA). Data are presented as mean $\pm \mathrm{SD}$, controlled for normal distribution by Kolmogorov-Smirnov test and compared using unpaired Student's t-test when normally distributed. Nonparametric tests were also applied in abnormal distribution (Mann-Whitney U test). Categorical data between two or more groups were compared by the Pearson $\chi^{2}$ test. The correlation of continuous variables was analyzed by Pearson and categorical variables by Spearman correlation analysis. Linear regression analysis was used to identify the independent predictors of cardiac fibrosis in patient with NDC. A probability value of $p<0.05$ was considered as significant.

\section{Results}

Study population included $5(23 \%)$ women and $17(77 \%)$ men. The age range was $27-74$ years (mean age: 47$)$. Seven (32\%) patients were in 


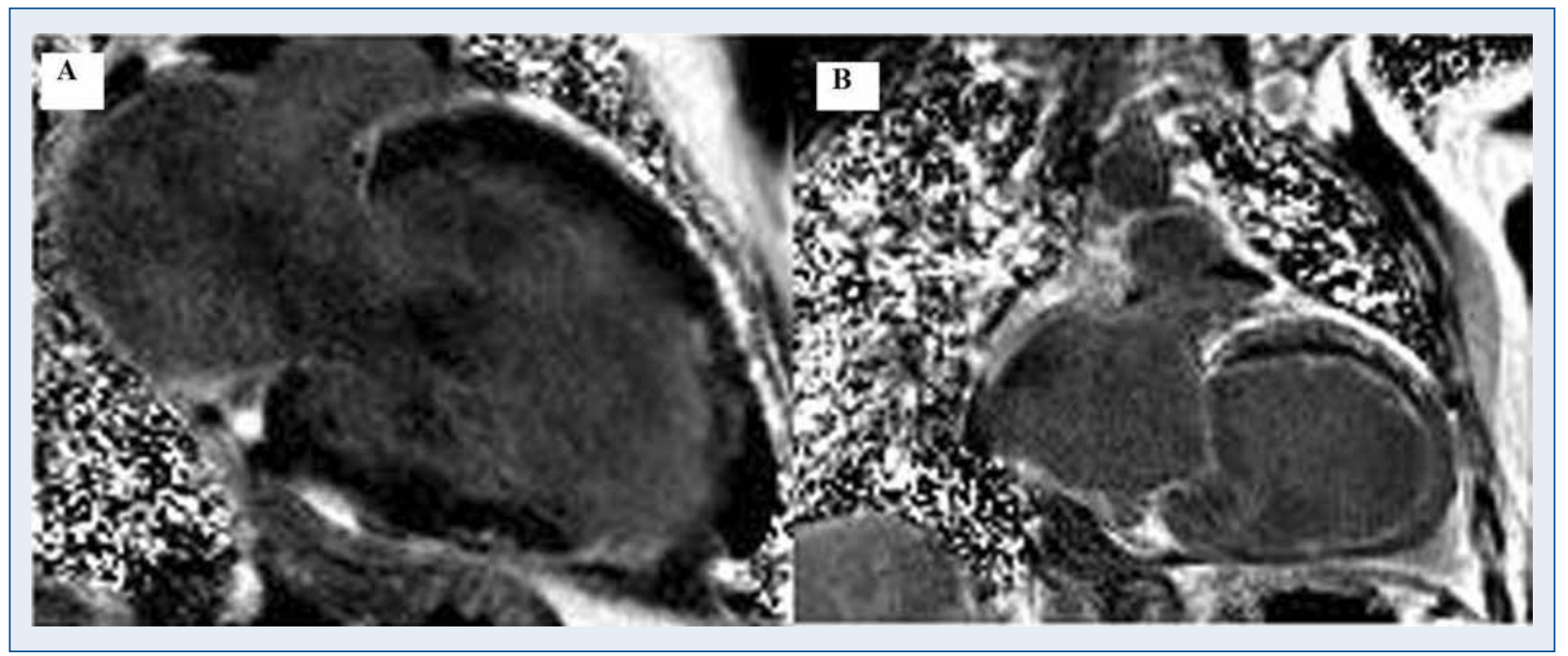

Figure 2. Apical (A) and extensive (B) subendocardial late gadolinium enhancement demonstrating cardiac fibrosis.

Table 1. Frequency of fibrosis amongst the left ventricular segments.

\begin{tabular}{lcccccc}
\hline & Inferoseptum & Anterolateral & Anterior & Inferior & $\begin{array}{c}\text { Antero- } \\
\text { septum }\end{array}$ & Posterolateral \\
\hline Basal & $0(0 \%)$ & $5(45.4 \%)$ & $2(18.1 \%)$ & $4(36.3 \%)$ & $2(18.1 \%)$ & $0(0 \%)$ \\
Midwall & $0(0 \%)$ & $5(45.4 \%)$ & $1(9 \%)$ & $6(54.5 \%)$ & $0(0 \%)$ & $1(9 \%)$ \\
Apical & $0(0 \%)$ & $2(18.1 \%)$ & $0(0 \%)$ & $3(27.2 \%)$ & & \\
\hline
\end{tabular}

Table 2. The clinical, ECG and biochemical properties of LGE- and LGE + groups.

\begin{tabular}{lccc}
\hline & LGE- group $(\mathbf{n}=\mathbf{1 1})$ & LGE+ group $(\mathbf{n}=\mathbf{1 1})$ & $\mathbf{P}$ \\
\hline Age [years] & $51.4 \pm 12.3$ & $43.2 \pm 10.9$ & 0.11 \\
Female gender [\%] & $3(27 \%)$ & $2(18 \%)$ & 0.61 \\
SBP/DBP [mm Hg] & $110 \pm 11 / 67 \pm 8$ & $110 \pm 15 / 77 \pm 6$ & 0.35 \\
NYHA class I-II/III-IV & $9 / 2$ & $6 / 5$ & 0.18 \\
NT-proBNP [pg/mL] & $1490 \pm 926$ & $1100 \pm 1043$ & 0.49 \\
PR duration [ms] & $205.1 \pm 34.0$ & $203.0 \pm 30.0$ & 0.26 \\
QRS duration [ms] & $123.2 \pm 28.6$ & $139.4 \pm 38.1$ & 0.33 \\
LBBB & $3(27 \%)$ & $2(18 \%)$ & 0.15 \\
\hline
\end{tabular}

LGE — late gadolinium enhancement; SBP/DBP — systolic/diastolic blood pressure; NYHA — New York Heart Association; NT-proBNP — $\mathrm{N}$-terminal pro-B-type natriuretic peptide; LBBB — left bundle branch block

NYHA class III or IV. Plasma NT-pro BNP levels ranged between $101 \mathrm{pg} / \mathrm{mL}$ and $2685 \mathrm{pg} / \mathrm{mL}$, and the median value was $726 \mathrm{pg} / \mathrm{mL}$. The mean $\mathrm{LVEF}$ of the study patients was $31 \pm 6.8 \%$. The mean functional MR volume was $18 \pm 9 \mathrm{~mL}$. Patients were divided into two groups according to the presence of LGE in CMR. Eleven (50\%) patients had LGE in CMR (Fig. 2). The most frequently involved cardiac segments were mid-inferior, mid-lateral, basal-lateral and basal inferior segments (Table 1). Multiple cardiac segments were involved in most of the patients $(\mathrm{n}=5 ; 45.4 \%)$. Linear subendocardial involvement was the most frequent type (42.7\%), followed by patchy foci transmural (31.8\%), mid-wall stria (19.6\%) and linear subepicardial (5.9\%) involvement consecutively.

LGE - and LGE + groups both included 11 patients ( $8 \mathrm{men} / 3$ women; mean age: $51 \pm 12$ years and 9 men/ 2 women; mean age: $43 \pm 10$ years, respectively). LGE- and LGE + patients were similar in terms of clinical, ECG and standard echocardiographic measurements (Tables 2, 3). 
Table 3. The echocardiographic properties of LGE- and LGE + groups.

\begin{tabular}{lccc}
\hline & LGE- group $(\mathbf{n}=\mathbf{1 1})$ & LGE+ group $(\mathbf{n}=\mathbf{1 1})$ & P \\
\hline LVESD [cm] & $5.41 \pm 0.89$ & $5.79 \pm 1.12$ & 0.38 \\
LVEDD [cm] & $6.37 \pm 0.91$ & $6.78 \pm 1.23$ & 0.39 \\
LA [cm] & $4.29 \pm 0.74$ & $4.40 \pm 0.75$ & 0.72 \\
LV dp/dt [mm Hg/s] & $638 \pm 83.7$ & $609.5 \pm 124.9$ & 0.08 \\
LVEF [\%] & $31.5 \pm 7.1$ & $30.2 \pm 6.7$ & 0.67 \\
E [m/s] & $0.78 \pm 0.35$ & $0.81 \pm 0.24$ & 0.83 \\
A [m/s] & $0.62 \pm 0.22$ & $0.64 \pm 0.24$ & 0.83 \\
E/A ratio & $1.53 \pm 1.05$ & $1.47 \pm 0.77$ & 0.87 \\
EDT [ms] & $203.7 \pm 53.9$ & $187.9 \pm 64.2$ & 0.53 \\
Sm [cm/s] & $3.35 \pm 1.09$ & $3.62 \pm 0.87$ & 0.53 \\
e' [cm/s] & $3.39 \pm 1.48$ & $4.03 \pm 1.33$ & 0.31 \\
RVs [cm/s] & $9.09 \pm 3.07$ & $9.5 \pm 2.44$ & 0.73 \\
MR vol. [mL] & $16.9 \pm 8.8$ & $19.0 \pm 10.3$ & 0.63 \\
E/e' ratio & $30.5 \pm 28.1$ & $22.3 \pm 10.8$ & 0.40 \\
\hline
\end{tabular}

LGE — late gadolinium enhancement; A - mitral inflow A wave velocity; E — mitral inflow E wave velocity; LVEF — left ventricular ejection fraction; EDT - mitral inflow E wave deceleration time; E/A - mitral inflow e wave/a wave ratio; LA - left atrium diameter; LV dP/ $\mathrm{dt}$ - left ventricular: delta pressure/delta time; LVEDD - left ventricular end diastolic diameter; LVESD - left ventricular end systolic diameter; RVs - tricuspid annulus peak systolic velocity by TDI; Sm — basal septum peak systolic velocity by TDI; $e^{\prime}$ - basal septum early diastolic velocity by TDI; MR vol. — mitral regurgitation volume

Table 4. The tissue Doppler and torsion properties of the patient groups.

\begin{tabular}{|c|c|c|c|}
\hline & LGE- group $(n=11)$ & LGE + group $(n=11)$ & $\mathbf{P}$ \\
\hline GLS [\%] & $-8.37 \pm 4.19$ & $-8.59 \pm 3.25$ & 0.89 \\
\hline GCS [\%] & $-6.84 \pm 4.25$ & $-8.44 \pm 3.77$ & 0.37 \\
\hline GRS [\%] & $19.6 \pm 12.3$ & $16.1 \pm 7.42$ & 0.43 \\
\hline Rot-basal [] & $-2.67 \pm 2.37$ & $-5.14 \pm 2.22$ & 0.02 \\
\hline Rot-apical $\left[{ }^{\circ}\right]$ & $5.16 \pm 2.96$ & $-0.10 \pm 4.37$ & 0.004 \\
\hline Twist $\left[{ }^{\circ}\right]$ & $7.83 \pm 3.03$ & $5.04 \pm 3.23$ & 0.05 \\
\hline Torsion $[\% \mathrm{~cm}]$ & $1.03 \pm 0.40$ & $0.62 \pm 0.44$ & 0.03 \\
\hline Untwisting rate $[\%$ s] & $-57.1 \pm 26.8$ & $-45.0 \pm 25.0$ & 0.28 \\
\hline Reversed apical rotation [\%] & $0(0 \%)$ & $5(45 \%)$ & 0.01 \\
\hline Reversed basal rotation [\%] & $1(0.9 \%)$ & $0(0 \%)$ & 0.31 \\
\hline
\end{tabular}

LGE — late gadolinium enhancement; GLS - global longitudinal strain; GCS — global circumferantial strain; GRS — global radial strain; Rot-basal - basal left ventricular rotation; Rot-apical — apical left ventricular rotation

Myocardial deformation and rotation parameters calculated by speckle tracking analysis are shown in Table 4. GLS, GCS, GRS values were similar between the two groups, whereas LV rotation parameters were significantly different. LGE + patients had significantly higher basal systolic rotation and significantly lower apical systolic rotation, twist and torsion when compared to the LGE- patients. However, untwisting rate was similar between the two groups. Torsion was significantly correlated with LVEF and MR-index (cardiac fibrosis index) $(\mathrm{r}=0.64, \mathrm{p}=0.04 ; \mathrm{r}=0.80, \mathrm{p}=0.005$, respectively $)$ (Figs. 3, 4). Linear regression analysis revealed that only MR-index (OR 1.53, 95\% CI 2.9-3.06, p = $=0.02$ ) was independent correlated factor of torsion.
Although 17 NDC patients had basal-clockwise and apical-counterclockwise systolic rotations (normally directed apical and basal rotation), interestingly, $5 \mathrm{LGE}+$ patients had reversed (clockwise) apical systolic rotation (Fig. 5), with significant difference in comparison to LGE- patients. However, one LGE- patient had also reversed (counterclockwise) basal systolic rotation but it was not statistically significant (Table 4). None of the patients exhibited reversed basal and reversed apical systolic rotation together. Accordingly, to further analyze the effect of apical motion direction, patients were divided into two subgroups with respect to the presence of reversed apical systolic rotation. Compared to the patients with normally 


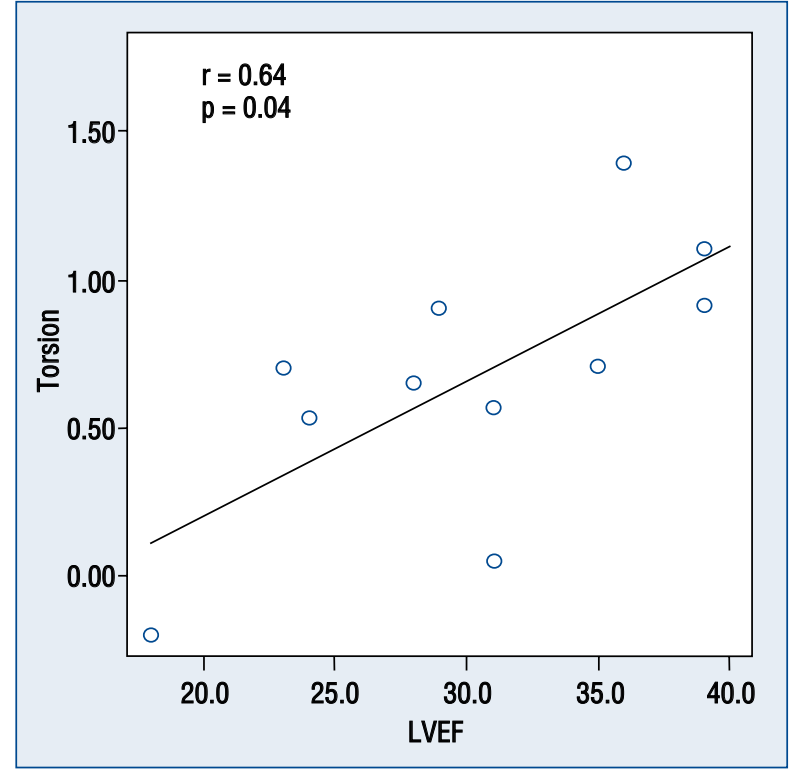

Figure 3. Correlation of torsion with left ventricular ejection fraction (LVEF).

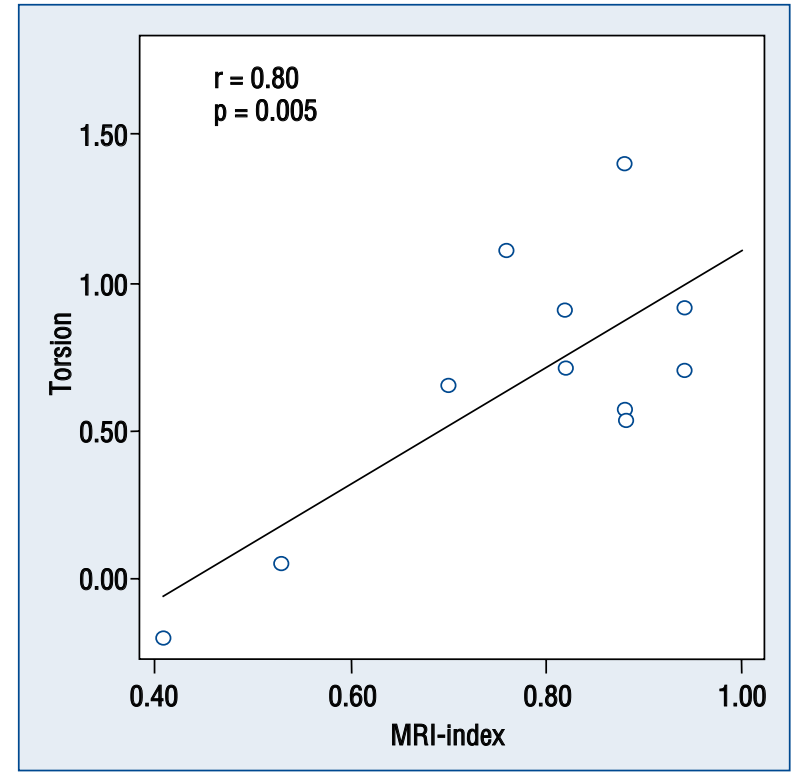

Figure 4. Correlation of torsion with cardiac fibrosis index (magnetic resonance imaging [MRI]-index).

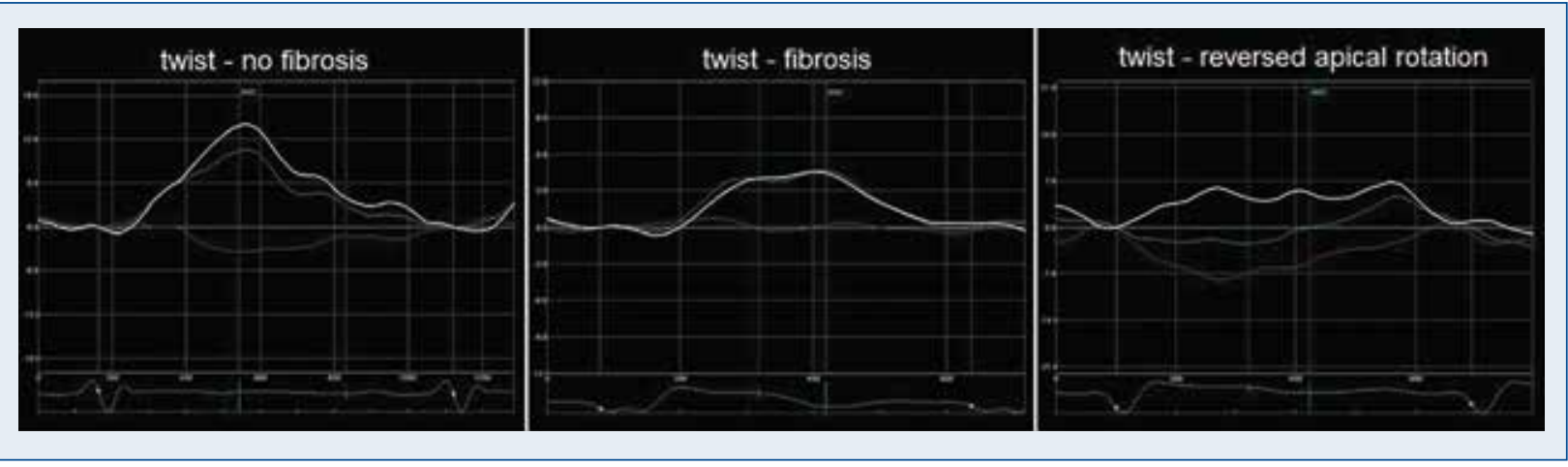

Figure 5. In patients with cardiac fibrosis (in the middle), apical systolic rotation, and thus, left ventricular twist is lower, in comparison to the patients without cardiac fibrosis (in the left). However in patients with extensive cardiac fibrosis, reversed (clockwise) apical systolic rotation is observed (in the right).

directed apical systolic rotation, those with reversed apical systolic rotation had similar NYHA functional class, conventional echocardiographic parameters, myocardial deformation parameters (GLS,GCS,GRS) and untwisting rate; but they had significantly greater NT-proBNP values $(1547 \pm$ $\pm 945 \mathrm{pg} / \mathrm{mL}$ vs. $389 \pm 303 \mathrm{pg} / \mathrm{mL}, \mathrm{p}=0.007)$, basal systolic rotation $\left(-6.78 \pm 1.21^{\circ}\right.$ vs. $-3.06 \pm$ $\left.\pm 2.2^{\circ}, \mathrm{p}<0.001\right)$, and significantly lower apical systolic rotation $\left(-3.71 \pm 3.64^{\circ}\right.$ vs. $4.36 \pm 2.81^{\circ}$, $\mathrm{p}<0.001)$, twist $\left(3.07 \pm 3.44^{\circ}\right.$ vs. $7.43 \pm 2.72^{\circ}$, $\mathrm{p}=0.04)$, torsion $\left(0.35 \pm 0.51^{\circ} / \mathrm{cm} v s .0 .95 \pm 0.38^{\circ} / \mathrm{cm}\right.$, $\mathrm{p}=0.05)$ and MR-index $(0.66 \pm 0.20$ vs. $0.95 \pm$ $\pm 0.06, p=0.03$ ) (Tables $5-7)$. Forty percent of patients ( 2 out of 5 ) with reversed apical systolic rotation and only $11.7 \%$ of patients ( 2 out of 17 ) with normally directed apical systolic rotation demonstrated LGE in at least one apical myocardial segment $(p=0.03)$ (Table 7). Amongst the apical myocardial segments, inferior-apical and anterolateral-apical segments were the most frequently affected ones.

\section{Discussion}

\section{Main findings of the study}

In this study we could demonstrate that the presence of cardiac fibrosis in NDC patients is 
Table 5. The clinical and biochemical properties of the patients with normally directed apical rotation and reversed apical rotation.

\begin{tabular}{|c|c|c|c|}
\hline & $\begin{array}{l}\text { Normally directed } \\
\text { apical rotation }(n=17)\end{array}$ & $\begin{array}{l}\text { Reversed apical } \\
\text { rotation }(n=5)\end{array}$ & $\mathbf{P}$ \\
\hline Age [years] & $46.6 \pm 12.9$ & $49.8 \pm 9.6$ & 0.57 \\
\hline Female gender [\%] & $3(17.6 \%)$ & $2(40 \%)$ & 0.30 \\
\hline SBP/DBP [mm Hg] & $114 \pm 9 / 63 \pm 3$ & $118 \pm 10 / 72 \pm 4$ & 0.73 \\
\hline NYHA class I-II/III-IV & $12 / 5$ & $3 / 2$ & 0.66 \\
\hline NT-proBNP [pg/mL] & $389 \pm 303$ & $1547 \pm 945$ & 0.007 \\
\hline
\end{tabular}

SBP/DBP — systolic/diastolic blood pressure; NYHA — New York Heart Association; NT-proBNP — N-terminal pro-B-type natriuretic peptide

Table 6. The echocardiographic properties of the patients with normally directed apical rotation and reversed apical rotation.

\begin{tabular}{lccc}
\hline & $\begin{array}{c}\text { Normally directed } \\
\text { apical rotation }(\mathbf{n}=\mathbf{1 7})\end{array}$ & $\begin{array}{c}\text { Reversed apical } \\
\text { rotation }(\mathbf{n}=\mathbf{5})\end{array}$ & $\mathbf{P}$ \\
\hline LVESD [cm] & $5.72 \pm 1.03$ & $5.21 \pm 0.89$ & 0.32 \\
LVEDD [cm] & $6.71 \pm 1.08$ & $6.12 \pm 1.07$ & 0.31 \\
LA [cm] & $4.45 \pm 0.73$ & $3.98 \pm 0.68$ & 0.22 \\
LV dp/dt [mm Hg/s] & $675.6 \pm 94.7$ & $571.2 \pm 158.1$ & 0.28 \\
EF [\%] & $30.8 \pm 6.5$ & $31.0 \pm 8.7$ & 0.97 \\
E $[\mathrm{m} / \mathrm{s}]$ & $0.78 \pm 0.31$ & $0.85 \pm 0.26$ & 0.64 \\
A [m/s] & $0.60 \pm 0.20$ & $0.74 \pm 0.30$ & 0.38 \\
E/A ratio & $1.52 \pm 0.92$ & $1.42 \pm 0.92$ & 0.84 \\
EDT [ms] & $196.4 \pm 47.2$ & $193.8 \pm 94.9$ & 0.95 \\
Sm $[\mathrm{cm} / \mathrm{s}]$ & $3.4 \pm 0.98$ & $3.76 \pm 1.01$ & 0.51 \\
$\mathrm{e}^{\prime}[\mathrm{cm} / \mathrm{s}]$ & $3.77 \pm 1.45$ & $3.57 \pm 1.41$ & 0.79 \\
RVs $[\mathrm{cm} / \mathrm{s}]$ & $9.34 \pm 2.73$ & $9.14 \pm 2.96$ & 0.89 \\
MR vol. [mL] & $18.5 \pm 10.1$ & $15.6 \pm 6.4$ & 0.49 \\
E/e' ratio & $26 \pm 22.8$ & $27 \pm 14.4$ & 0.90 \\
\hline
\end{tabular}

A - mitral inflow A wave velocity; E - mitral inflow E wave velocity; LVEF — left ventricular ejection fraction; EDT - mitral inflow E wave deceleration time; E/A — mitral inflow e wave/a wave ratio; LA — left atrium diameter; LV dP/dt — left ventricular: delta pressure/delta time; LVEDD - left ventricular end diastolic diameter; LVESD — left ventricular end systolic diameter; RVs - tricuspid annulus peak systolic velocity by TDI; Sm — basal septum peak systolic velocity by TDI; $\mathrm{e}^{\prime}$ — basal septum early diastolic velocity by TDI; MR vol. — mitral regurgitation volume

Table 7. The tissue Doppler and rotation parameters of the patients with normally directed apical rotation and reversed apical rotation.

\begin{tabular}{|c|c|c|c|}
\hline & $\begin{array}{c}\text { Normally directed } \\
\text { apical rotation }(n=17)\end{array}$ & $\begin{array}{l}\text { Reversed apical } \\
\text { rotation }(\mathrm{n}=5)\end{array}$ & $\mathbf{P}$ \\
\hline GLS [\%] & $-8.20 \pm 3.73$ & $-9.64 \pm 3.72$ & 0.52 \\
\hline GCS [\%] & $-7.16 \pm 4.15$ & $-9.50 \pm 3.09$ & 0.25 \\
\hline GRS [\%] & $18.3 \pm 11.2$ & $16.2 \pm 4.1$ & 0.54 \\
\hline Rot-basal $\left[^{\circ}\right]$ & $-3.06 \pm 2.2$ & $-6.78 \pm 1.21$ & $<0.001$ \\
\hline Rot-apical $\left[{ }^{\circ}\right]$ & $4.36 \pm 2.81$ & $-3.71 \pm 3.64$ & $<0.001$ \\
\hline Twist $\left[{ }^{\circ}\right]$ & $7.43 \pm 2.72$ & $3.07 \pm 3.44$ & 0.04 \\
\hline Torsion $[\% / \mathrm{cm}]$ & $0.95 \pm 0.38$ & $0.35 \pm 0.51$ & 0.05 \\
\hline Untwisting rate $[\%]$ & $-49.08 \pm 25.6$ & $-57.9 \pm 29.3$ & 0.56 \\
\hline MR-index [\%] & $0.95 \pm 0.06$ & $0.66 \pm 0.20$ & 0.03 \\
\hline Apical fibrosis [\%] & $2(11.7 \%)$ & $2(40 \%)$ & 0.03 \\
\hline
\end{tabular}

GLS - global longitudinal strain; GCS - global circumferantial strain; GRS - global radial strain; MR-index - fibrosis index in cardiac magnetic resonance; Rot-basal — basal left ventricular rotation; Rot-apical — apical left ventricular rotation 
related to decreased or even reversed apical systolic rotation, as well as decreased twist and torsion. Furthermore, reversed apical systolic rotation with consequent loss of torsion reflected a more advanced disease stage with more severe cardiac fibrosis (significantly lower MR-index), more severe systolic dysfunction and markedly increased NT-proBNP in comparison to normally directed apical rotation. Cardiac fibrosis was most frequently detected in mid-inferior, mid-lateral, basal-lateral and basal-inferior segments in our study population.

\section{Left ventricular function and rotational dynamics}

The twisting motion of the LV around its long axis results from the contraction of the obliquely oriented epicardial and endocardial fibers. LV myofibers geometry changes smoothly from a slightly oblique orientation in the subendocardium to a circumferential orientation in the midwall and again to an oblique orientation in the subepicardium $[23,24]$. Because of this typical orientation, when viewed from the apex, the $\mathrm{LV}$ performs a clockwise rotation at the base and counterclockwise rotation at the apex, resulting in LV twist. LV twist is a critically important mechanism for the efficiency of LV function. $\mathrm{LV}$ apical rotation represents the main determinant of global LV systolic twist and torsion, resulting in storage of potential energy during systole, while rapid apical back rotation with sudden untwisting during isovolumic relaxation plays an important role in the suction of blood into the $\mathrm{LV}$, generating intraventricular pressure gradients for LV diastolic filling [25, 26]. In our study, the amplitude of LV systolic rotation at basal level was increased while apical systolic rotation, twist and torsion were reduced in NDC patients with cardiac fibrosis compared with LGE-patients. There was also significant correlation between LV torsion and degree of systolic dysfunction (LVEF). Moreover, we detected reversed systolic apical rotation reflecting more severely impaired LV rotational dynamics and LV systolic performance in patients with cardiac fibrosis when compared to those with normally directed apical rotation. These changes in the pattern of apical rotation could be explained by increased $L V$ sphericity leading to widening of the apex. This results in the loss of the oblique architecture of the ascending and descending apical loop fibers which become more transverse and more closely resemble the horizontal fiber orientation of the basal loop. In the end, progressive LV dilation and increased sphericity lead to changes in myofibre orientation and in torsional dynamics, triggering further deterioration of systolic and diastolic function.

\section{Myocardial fibrosis and rotational dynamics}

Patients with heart failure often show varying patterns of scarring and myocardial fibrosis. These regions of myocardial fibrosis have been visualized by CMR, appearing as areas of LGE [11]. Cardiovascular magnetic resonance is a rapidly evolving technology that offers a comprehensive assessment of heart failure patients and is now the gold standard imaging modality to assess myocardial anatomy and viability. Besides, LGE by CMR (LGE-CMR) strongly predicts cardiac adverse outcomes [12]. It can be classified as 'ischemic type' and 'non-ischemic type'. Ischemic type is either subendocardial or transmural and is located in a region which consists with the perfusion territory of an epicardial coronary artery. The second type (non-ischemic) is patchy or linear stria of enhancement limited to the mid-myocardium of the ventricular wall [27]. Linear midwall hyperenhancement is usually found in the interventricular septum and is observed more frequently in patients with possible prior history of myocarditis. This confirms that the mechanisms of cardiac fibrosis formation are completely different in ischemic and nonischemic cardiomyopathies.

The mechanisms for cardiac fibrosis are thought to be the result of a combination of factors including genetic predisposition, exposure to toxins and pathogens, microvascular ischemia, and abnormal responses of immune and metabolic pathways like renin-angiotensin -aldosteron system [28, 29]. Case reports exhibit containing myocardial midwall fibrosis in familial conditions such as muscular dystrophy [30]. Exposure to pathogens such as viruses triggers fibrosis. Early CMR imaging of patients with acute myocarditis shows characteristic epicardial or midwall late enhancement in the acute phase [31], which may persist and subsequently develop into dilated cardiomyopathy. Histopathological studies, in this early phase, confirm fibrosis and inflammatory exudates, while patients with end stage DCM show interstitial fibrosis in the absence of any inflammation [28]. Myocardial fibrosis is also associated with adverse ventricular remodelling leading to the development of the heart failure and dilated cardiomyopathy in animal and human studies [28, 29]. During the development of $\mathrm{LV}$ adverse remodeling, the size and shape of the LV change in patients with DCM and the enlarged ventricle exhibits a spherical configuration. Thus, 
marked changes in LV rotational dynamics would be expected in this setting. In our NDC population, we demonstrated that myocardial torsion was correlated with cardiac fibrosis index, defined by LGE-CMR, and with LVEF. Furthermore, we found that reversed apical rotation group exhibited significantly higher frequency of apical segment fibrosis in comparison to the normally directed apical rotation group. Accordingly, we conclude that cardiac fibrosis may be the main reason for disturbed apical rotation, twist and torsion in NDC patients. The presence of more extensive cardiac fibrosis (or MR-index) in patients with reversed apical rotation and with complete loss of torsion, seems like supporting this result.

\section{Clinical implications}

The findings of our study highlight the impact of cardiac fibrosis on apical and basal rotation, twist and torsion dynamics in failing left ventricles. This may underscore the potential clinical benefits of cardiac fibrosis index, defined by LGE-CMR, for certain therapeutic procedures such as cardiac resynchronization therapy (CRT). Thus, in the future, cardiac fibrosis index may be a useful parameter to indicate LV dyssynchrony and to select CRT candidates, which needs further investigations.

As we found reversed apical rotation in some of our NDC patients, revealing more severe LV disease with extensive cardiac fibrosis, this rotational parameter could be useful marker to be assessed before and after CRT. Whether reversed apical rotation can identify responders to CRT remains to be tested.

\section{Limitations of the study}

A technical limitation was that speckle tracking echocardiography is highly dependent on frame rates, as well as image quality. Especially apical rotation analysis requires good apical image quality. Therefore, we tried to keep frame rates optimal during image acquisitions and a small number of subjects with insufficient image quality were not included at the beginning. Measuring apical rotation from certain anatomical point also seems controversial. In this study, measurements were done at the most distal level that did not show luminal closure during systole [32].

Another limitation of our study was the relatively small extent of patient population which could have compromised our ability to detect the relation between cardiac fibrosis and rotational dynamics. Although our results clearly demon- strated this relation, using larger sample size, we could have obtained more significant associations, more explanatory data underlying mechanisms of reversed apical rotation and its potential role in certain cardiac therapeutic modalities. However, the reason was the selection of highly specific patient group, with good image quality.

\section{Conclusions}

Cardiac fibrosis identified by LGE-CMR is closely related to the myocardial rotational dynamics in patients with NDC. Cardiac fibrosis index was correlated with myocardial torsion and LV systolic function and may be used for the evaluation of cardiac condition. Reversed apical systolic rotation indicated more severe myocardial disease with more extensive cardiac fibrosis and may be useful parameter to predict response to CRT. But since we didn't have significant number of patient implanted CRT, we could not be able to assess this relation and further studies will be required.

\section{Conflict of interest: none declared}

\section{References}

1. Pitt B, Zannad F, Remme WJ et al. The effect of spironolactone on morbidity and mortality in patients with severe heart failure. Randomized Aldactone Evaluation Study Investigators. N Engl J Med, 1999; 341: 709-717.

2. Zannad F, Briancon S, Juilliere Y et al. Incidence, clinical and etiologic features, and outcomes of advanced chronic heart failure: The EPICAL study. J Am Coll Cardiol, 1999; 33: 734-742.

3. Solomon SD, Anavekar N, Skali H et al. Influence of ejection fraction on cardiovascular outcomes in a broad spectrum of heart failure patients. Circulation, 2005; 112: 3738-3744.

4. Uriel N, Torre-Amione G, Milo O et al. Echocardiographic ejection fraction in patients with acute heart failure: Correlations with hemodynamic, clinical, and neurohormonal measures and short-term outcome. Eur J Heart Fail, 2005; 7: 815-819.

5. Sengupta PP, Khandheria BK, Narula J. Twist and untwist mechanics of the left ventricle. Heart Fail Clin, 2008; 4: 315-324.

6. Korinek J, Wang J, Sengupta PP et al. Two-dimensional strain - A Doppler-independent ultrasound method for quantitation of regional deformation: Validation in vitro and in vivo. J Am Soc Echocardiogr, 2005; 18: 1247-1253.

7. Wang J, Khoury DS, Yue Y, Torre-Amione G, Nagueh SF. Left ventricular untwisting rate by speckle tracking echocardiography. Circulation, 2007; 116: 2580-2586.

8. Amundsen BH, Helle-Valle T, Edvardsen T et al. Noninvasive myocardial strain measured by speckle tracking echocardiography. Validation against sonomicrometry and tagged magnetic resonance imaging. J Am Coll Cardiol, 2006; 47: 789-793.

9. Notomi Y, Setser RM, Shiota T et al. Assessment of left ventricular torsional deformation by Doppler tissue imaging: Validation study with tagged magnetic resonance imaging. Circulation, 2005; 111: 1141-1147. 
10. McCrohon JA, Moon JC, Prasad SK et al. Differentiation of heart failure related to dilated cardiomyopathy and coronary artery disease using gadolinium-enhanced cardiovascular magnetic resonance. Circulation, 2003; 108: 54-59.

11. Assomull RG, Prasad SK, Lyne J et al. Cardiovascular magnetic resonance, fibrosis and prognosis in dilated cardiomyopathy. J Am Coll Cardiol, 2006; 48: 1977-1985.

12. Wu KC, Weiss RG, Thiemann DR et al. Late gadolinium enhancement by cardiovascular magnetic resonance heralds an adverse prognosis in nonischemic cardiomyopathy. J Am Coll Cardiol, 2008; 51: 2414-2421.

13. Schiller NB, Shah PM, Crawford M et al. Recommendations for quantitation of the left ventricle by two-dimensional echocardiography: American Society of Echocardiography Committee on Standards, Subcommittee on Quantitation of the Two-Dimensional Echocardiograms. J Am Soc Echocardiogr, 1989; 2: 358-367.

14. Werner GS, Schaefer C, Dirks R, Figulla HR, Kreuzer H. Prognostic value of Doppler echocardiographic assessment of left ventricular filling in idiopathic dilated cardiomyopathy. Am J Cardiol, 1994; 73: 792-798.

15. Enriquez-Sarano M, Miller FA Jr, Hayes SN, Bailey KR, Tajik AJ, Seward JB. Effective mitral regurgitant orifice area: Clinical use and pitfalls of the proximal isovelocity surface area method. J Am Coll Cardiol, 1995; 25: 703-709.

16. Bargiggia GS, Bertucci C, Recusani F et al. A new method for estimating left ventricular $\mathrm{dP} / \mathrm{dt}$ by continuous wave Doppler-echocardiography. Validation studies at cardiac catheterization. Circulation, 1989; 80: 1287-1292.

17. Yu CM, Wang Q, Lau CP et al. Reversible impairment of left and right ventricular systolic and diastolic function during short-lasting atrial fibrillation in patients with an implantable atrial defibrillator: A tissue Doppler imaging study. Pacing Clin Electrophysiol, 2001; 24: 979-988.

18. Yu CM, Lin H, Yang H, Kong SL, Zhang Q, Lee SW. Progression of systolic abnormalities in patients with "isolated" diastolic heart failure and diastolic dysfunction. Circulation, 2002; 105: 1195-1201.

19. Popescu BA, Beladan CC, Calin A et al. Left ventricular remodelling and torsional dynamics in dilated cardiomyopathy: Reversed apical rotation as a marker of disease severity. Eur J Heart Fail, 2009; 11: 945-951.

20. Cerqueira MD, Weissman NJ, Dilsizian V et al. Standardized myocardial segmentation and nomenclature for tomographic imaging of the heart: A statement for healthcare professionals from the Cardiac Imaging Committee of the Council on Clinical
Cardiology of the American Heart Association. Circulation, 2002; 105: 539-542.

21. Kaandorp TA, Bax JJ, Schuijf JD et al. Head-to-head comparison between contrast-enhanced magnetic resonance imaging and dobutamine magnetic resonance imaging in men with ischemic cardiomyopathy. Am J Cardiol, 2004; 93: 1461-1464.

22. Tigen K, Karaahmet T, Kirma $\mathrm{C}$ et al. Diffuse late gadolinium enhancement by cardiovascular magnetic resonance predicts significant intraventricular systolic dyssynchrony in patients with non-ischemic dilated cardiomyopathy. J Am Soc Echocardiogr, 2010; 23: 416-422.

23. Greenbaum RA, Ho SY, Gibson DG, Becker AE, Anderson RH. Left ventricular fibre architecture in man. Br Heart J, 1981; 45: 248-263.

24. Ingels NB Jr, Hansen DE, Daughters GT $2^{\text {nd }}$, Stinson EB, Alderman EL, Miller DC. Relation between longitudinal, circumferential, and oblique shortening and torsional deformation in the left ventricle of the transplanted human heart. Circ Res, 1989; 64: 915-927.

25. Opdahl A, Helle-Valle T, Remme EW et al. Apical rotation by speckle tracking echocardiography: a simplified bedside index of left ventricular twist. J Am Soc Echocardiogr, 2008; 21: 1121-1128.

26. Notomi Y, Martin-Miklovic MG, Oryszak SJ et al. Enhanced ventricular untwisting during exercise: A mechanistic manifestation of elastic recoil described by Doppler tissue imaging. Circulation, 2006; 113: 2524-2533.

27. McCrohon JA, Moon JC, Prasad SK. Differentiation of heart failure related to dilated cardiomyopathy and coronary artery disease using gadolinium enhanced cardiovascular magnetic resonance. Circulation, 2003; 108: 54-59.

28. Ryoke T, Gu Y, Mao L et al. Progressive cardiac dysfunction and fibrosis in the cardiomyopathic hamster and effects of growth hormone and angiotensin-converting enzyme inhibition. Circulation, 1999; 100: 1734-1743.

29. Heling A, Zimmermann R, Kostin S et al. Increased expression of cytoskeletal, linkage, and extracellular proteins in failing human myocardium. Circ Res, 2000; 86: 846-853.

30. Varghese A, Pennell DJ. Late gadolinium enhanced cardiovascular magnetic resonance in Becker muscular dystrophy. Heart, 2004; 90: e59.

31. Mahrholdt H, Goedecke C, Wagner A et al. Cardiovascular magnetic resonance assessment of human myocarditis: A comparison to histology and molecular pathology. Circulation, 2004; 109: 1250-1258.

32. Notomi Y, Lysyansky P, Setser RM et al. Measurement of ventricular torsion by two-dimensional ultrasound speckle tracking imaging. J Am Coll Cardiol, 2005; 45: 2034-2041. 